\title{
Optimized Broadcast Scheme for Mobile Ad hoc Networks
}

\author{
Ahmed Amari ${ }^{1}$, Nadjib Achir ${ }^{1,2}$, Paul Muhlethaler ${ }^{1}$, and Anis Laouiti ${ }^{3}$ \\ ${ }^{1}$ INRIA HiPERCOM, Rocquencourt B.P. 105, 78153 Le Chesnay, \\ France \\ ${ }^{2}$ L2TI - Institut Galilée - University Paris 13 \\ ${ }^{3}$ Telecom SudParis, Evry, France
}

January 21, 2017

\begin{abstract}
In this paper we propose an optimized broadcasting mechanism which uses very limited signaling overhead. The main objective is to select the most appropriate relay nodes according to a given cost function. Basically, after receiving a broadcast packet each potential relay node computes a binary code according to a given cost function. Then, each node starts a sequence of transmit/listen intervals following this code. In other words, each 0 corresponds to a listening interval and each 1 to a transmit interval. During this active acknowledgment signaling period, each receiver applies the following rule: if it detects a signal during any of its listening intervals, it quits the selection process, since a better relay has also captured the packet. Finally, we split the transmission range into several sectors and we propose that all the nodes within the same sector use the same CDMA orthogonal spreading codes to transmit their signals. The CDMA codes used in two different sectors are orthogonal, which guarantees that the packet is broadcast in all possible directions.
\end{abstract}

\section{Introduction}

Mobile ad hoc networks (MANETs) enable mobile nodes to communicate with each other without the need for a preexisting infrastructure. Since the radio range of each node is limited, multi-hop routing protocols are required to allow nodes that are not within range of each other to communicate. In this case, all the nodes need to act not only as simple nodes but also as routers to forward packets to the next hop towards the destination. Pointto-point communication is not the only communication mode, broadcast 
communication is also very important and is prominent in many applications. Moreover, broadcast is widely used for a large number of routing protocols proposed in the literature. Indeed, many of existing routing protocols use the easiest broadcasting scheme, the simple flooding algorithm, to construct the routing tables, such as in OLSR protocol. Unfortunately, many studies pointed out the fact that pure flooding may lead to many contentions and collisions, and thus serious performance degradations. This situation is known as the "broadcast-storm" problem.

In order to cope with these issues many challenges need to be faced to provide efficient broadcasts over mobile ad hoc networks. In this paper we focus on one of the main challenges, which is how to select the most appropriate forwarding nodes. Basically, classical flooding scheme can be improved if only a few nodes forward the messages sent by the initiator of the broadcast. More precisely, the main challenge is to find and select the fewest number of nodes to forward a message while maximizing the coverage. This problem has been defined in the literature as the problem of finding the minimum Connected Dominating Set (CDS). Unfortunately, finding this solution is NP-hard [1].

Here we propose an optimized broadcasting mechanism which uses a very limited overhead. Our broadcasting algorithm uses an advanced acknowledgment scheme to select the appropriate relay nodes from all the potential relays within the transmission range. We propose that each node that captured a packet is required to transmit a short acknowledgment made up of signaling bursts. This sequence is computed by each node according to a given cost function. The acknowledgment is used to select the best potential relay nodes and consists of a sequence of intervals of the same length in which a given receiver can either transmit or listen. During this active acknowledgment signaling period, if a receiver detects a signal during any of its listening intervals, it quits the selection process, since it indicates that a better relay has also captured the packet. We thus obtain a powerful selection process to determine the most appropriate relay nodes. Finally, to distribute the packet in all possible directions, we propose that the transmission area is split into several sectors. Moreover, to guarantee that one relay is selected in each sector, a different CDMA spreading code is used in each sector. In two different sectors CDMA spreading codes are orthogonal.

The rest of this paper is organized as follows: In section 2 we provide the state of the art. In Section 3 we detail the protocol we propose. Simulation parameters, metrics, and results obtained with our broadcasting scheme are presented in Section 4. Finally, in Section 5 we conclude the paper, and suggest future research directions. 


\section{State of the Art}

Several mechanisms have been proposed in the literature in order to optimize the broadcast transmissions in MANETs, in terms of reliability, efficiency, energy consumption and ability to satisfy the application QoS constraints. Assessment of the proposed schemes have been conducted for several applications like video or audio streaming, file sharing, communications for military applications or for civilians through wireless sensor networks [2] [3] [4] [5] [6]. Williams and Camp [7] categorize the broadcasting schemes into four families: simple flooding, probability based methods, area based methods and neighborhood based methods.

The first family, simple flooding, is considered as the default solution for packet broadcasting in ad hoc networks. In this scheme, each node needs to retransmit each received packet to its neighbors once. Since packets are duplicated at each node simple flooding ensures that every node in the network receives each broadcast packet at least once. Moreover no signaling is needed. Unfortunately, when considering high density networks, Ni et al. [2] show that simple flooding may lead to serious message redundancy, a high level of contention and many collisions. This situation is often referred to the "broadcast storm" problem.

The second family, probability based methods, includes two broadcasting schemes [2]: probability based and counter based. In probability based scheme, each node is affected a predefined packet re-broadcasting probability: $p$. Whenever a node receives a packet, it will rebroadcast it with probability $p$, or drop it with a probability: $1-p$. Note, that if $p=1$ then this scheme leads to simple flooding. In the second scheme, counter based, each node rebroadcasts a given packet if the same copies of this packet have already been received less than a given number of times. Thus, compared to previous broadcasting schemes, the reduction in the number of redundant broadcast packets that could be obtained by the counter based scheme will rely on the network density to ensure the packet delivery. In a dense MANET, few nodes will rebroadcast packets, while with a sparse topology, a majority of nodes will have to rebroadcast the packets.

In the third family, area based methods, the retransmission of a packet is decided according to the estimation of the relative physical distance between the transmitter and its relay. Within this family, we can cite two schemes [2]: distance based and location based. In the first scheme, each node is given a predefined distance threshold $(d)$. When a node receives a broadcast packet it estimates its physical distance from the last relaying node. If the distance is less than the threshold, the packet is then drooped. Otherwise, the packet is retransmitted. The main problem with this scheme concerns accurately assessing this distance. For instance, the distance can be estimated from the measured signal strength of the received packet. Unfortunately, this technique does not operate well in a realistic ad hoc network. In location 
based schemes, each node adds its location information in the header of each packet sent. Thus, packet retransmission is done only if the additional coverage area added by the receiver is greater than a given threshold.

Finally, the fourth family contains neighborhood based methods which exploit information on the neighborhood of the nodes. The main schemes belonging to this family are: self pruning [3], dominant pruning [3], scalable broadcasting [4], multipoint relay [5], Ad Hoc broadcast protocol [6] and Simplified Multicast Forwarding for MANETs [8]. In self pruning, each node piggybacks, into each retransmitted packet, its adjacent nodes. Thus, nodes that received this packet can check if their own adjacent nodes are the same as those piggybacked in the received packet. If so, the packet is dropped. Otherwise, it is re-transmitted. While self pruning exploits only one-hop node information, the remaining schemes dominant pruning, scalable broadcasting, multipoint relay, Ad Hoc broadcast protocol and Simplified Multicast Forwarding for MANETs require that all nodes have knowledge of their two-hop neighbors. Unfortunately, all neighborhood based methods require extra transmission overhead, especially in dense MANETs.

Recently Blaszczyszyn et al. [9] propose an opportunistic broadcast protocol for VANET. This protocol is very similar to our approach since it uses an active signaling acknowledgment to select the best relay among all the vehicles that have received the packet. Unfortunately, this algorithm was proposed only in the case of one dimension corresponding to roads.

\section{Proposed Algorithm}

As introduced earlier, the problem we study in this paper is, given an input MANET, how to determine the most adequate relays with minimum/maximum cost, according to a given cost function, in order to provide an efficient broadcast strategy. To meet this requirement, our idea is, as for unicast communications, to use an acknowledgment scheme to select the appropriate relay nodes from all potential relays within the transmission area. More precisely, we propose that each node that captured a packet is required to transmit a short acknowledgment made up of active signaling bursts. The idea of active signaling bursts was initially introduced in the HiPERLAN type 1 standard [10], where the objective is to merge priority contention and collision detection into an efficient channel access mechanism. Basically, each node willing to access the medium has to transmit access pattern of non-data signals in a preamble to the packet transmission attempts .

In HiPERLAN active signaling, each node must access the medium by transmitting an "access pattern" of non-data signals in the preamble to the packet. This access pattern basically consists of listening and signaling periods. The signal transmitted in the signaling period contains no data at 
all. The procedure of collision detection and conflict resolution is performed during the packet access preamble where the node alternates between listening and transmitting. The access rule in the access pattern is that when a node is in a listening period, if it detects some energy from another node, then it immediately stops the access scheme and defers its packet transmission for a later attempt. Otherwise the nodes transmit their packet at the end of the access pattern transmission. In this case, when a transmission attempt starts, each node in competition selects a new access pattern. Since this access pattern has a random component, the probability of two nodes having the same access pattern is reduced. The access patterns also depend on the access priority assigned to the packet by the MAC level data transfer service. Thus, it is clear that the node which has priority to transmit its packet is the one holding the pattern which presents the largest digital number expanded in binary, if we consider that one codes the pattern time slots with a 1 corresponding to a transmit period and 0 corresponding to a listening period.

Our proposed acknowledgment active signaling burst is quite similar to the HiPERLAN active signaling scheme, except that we propose to use it as an acknowledgment. We propose that each node receiving a packet has to transmit a short acknowledgment. In this case, several acknowledgments could be sent at the same time by all the nodes capturing the packet. In classical communication, this operation leads to several collisions between the acknowledgments, but not in our case. In fact each acknowledgment, denoted as a burst in the following, is not carrying any data at all but it consists of a sequence of intervals of the same length in which a given receiver can either transmit or listen. During the transmitting period each node sends a signal on the medium and during a listening period each node listens on the medium. These signaling bursts can be represented by binary sequences denoted in the following as burst codes. 0 denotes a listening interval and 1 denotes a transmitting interval. In the rest of this paper, we fix the size of the burst code to $L$ bits

We propose to use the following relay selection rule: if a receiver detects a signal (energy) during any of its listening intervals, it quits the selection process. This means that the receiver stops transmitting during the entire remaining part of the burst. We consider that the detection of a transmission during a listening interval implies that a better relay has also captured the data packet. When a node receives a data packet it computes its own burst code as a function of the criterion that we wish to optimize to select our "best" relay. The only condition that we need to satisfy is that the better the relay is, the higher the burst code is. For instance, if we consider that the best relays are the farthest nodes, we should code the distance separating the source node and the relay nodes in base 2 ; this distance in base 2 will be the burst code. Thus, we can easily check that the selection mechanism will always select the relay nodes having the highest burst code (i.e. the greatest 
distance), since there will always be an interval in which another relay with a smaller burst code (i.e. a smaller distance) listens when the relay with the highest burst code is transmitting. Finally, in order to discriminate between nodes using the same burst code (e.g. at the same distance from the transmitter) we add $r$ bits randomly selected.

In Figure 1, we represent an example of the selection process of three potential relays, $a, b$ and $c$. We can notice that node $a$, with a higher burst code wins the selection process. In this case, only node $a$ will relay the packet.

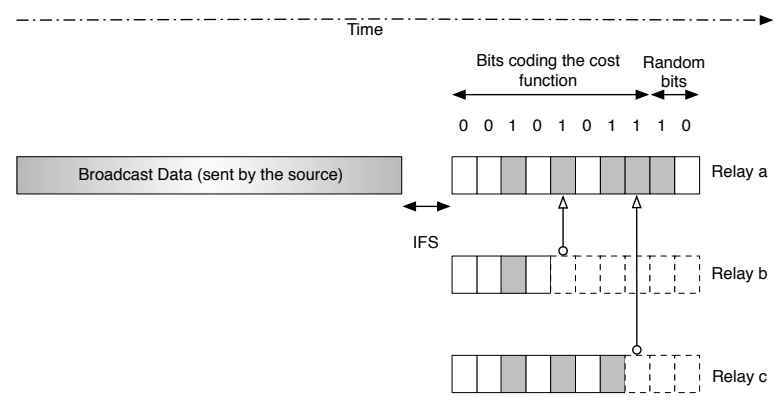

Figure 1: Selection process

Unfortunately, considering only this selection mechanism does not guarantee that the packet will be propagated in all possible directions. In addition to the acknowledgment signaling mechanism, we propose that the transmission area of each node is split into a given number of sectors, noted as $k$. The objective is that at least one node in each sector has to relay the packet. On the one hand, if we consider the whole transmission range as one unique sector $(k=1)$, the packet is relayed by only a few nodes. This may negatively impact the performance. On the other hand, having a large number of sectors will also affect the performance by increasing the number of packet collisions. The number of sectors should not be too small nor too large. Moreover, since we want at least one node to act as a relay node in each sector, we also need to guarantee that nodes belonging to adjacent sectors will not eliminate each other. In other words, the relay node selection process should act only between nodes belonging to the same sector. To achieve this requirement, one possible approach is to perform a sequential selection process phase, starting from the first sector up to the last sector. This approach will however increase the delay of the acknowledgment phase and thus reduce the network throughput. The solution that we propose is that during the transmitting period (corresponding to 1 in the burst code) each node must send a CDMA spreading code. The code that must be used by each potential relay is selected according to its sector.

CDMA orthogonal spreading codes are one of the major elements within 
the whole CDMA system. Essentially, the concept of CDMA is based around the fact that a data sequence is multiplied by a spreading code or sequence, which increases the bandwidth of the signal. Then, in the receiver, the same spreading code or sequence is used to extract the required data. Only when the required code is used, does the required data appear from the signal. In our case, we use the same principle, since each potential relay node has to multiply the burst code by the spreading code associated to its sector (denoted in the following as the sector code). If a potential relay is in listening mode, it must compute the autocorrelation between the received signal and its sector code. If the autocorrelation is equal to 1 , then this means that another node, belonging to the same sector, is in its transmitting period. This implies that a better potential relay has also captured the data packet and the node must quit the selection process.

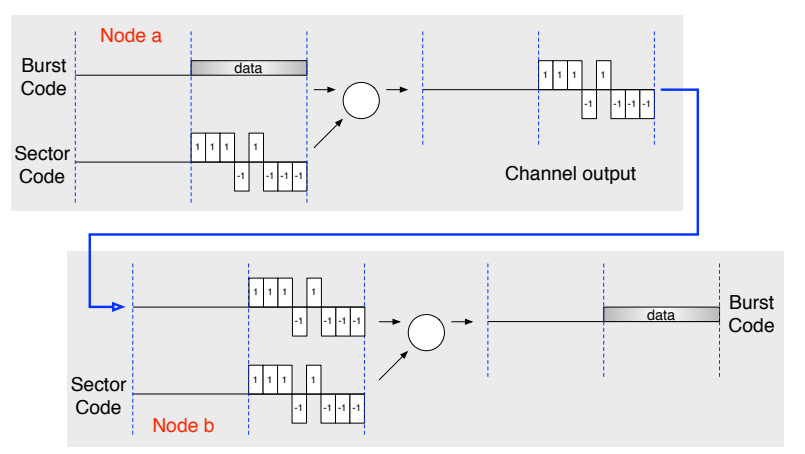

Figure 2: Selection process: two nodes in the same sector

In order to illustrate the proposed mechanism, let us consider the following two examples. In the first example, Figure 2, we illustrate the acknowledgment phase for one potential relay $a$ having a burst code equal to [0 1 . Here, this node has to listen to the medium during the first interval and transmit during the second interval. We also assume that this node is in a sector having [ $\left[\begin{array}{llllllll}1 & 1 & 1 & -1 & 1 & -1 & -1 & -1\end{array}\right]$ as its sector code. As illustrated, the node multiplies the burst code to the sector code and transmits the result on the channel. At the same time, we assume that a second node (node $b$ ) having a burst code equal to [0 0 ] belongs to the same sector as $a$. In this case, $b$ is listening during the two intervals. As we can see, node $b$ receives a signal during the second interval and computes the autocorrelation between the received signal and the code of its sector. If the result obtained is equal to 1 , which is the case in this example, than the node quits the selection process.

In the second example, Figure 3, we consider the case where two nodes belonging to two adjacent sectors receive the acknowledgment signals. As in the previous example, we assume that node $a$ has a burst code equal to [0 1] and a sector code equal to $\left[\begin{array}{llllllll}1 & 1 & 1 & -1 & 1 & -1 & -1 & -1\end{array}\right]$. We consider here 


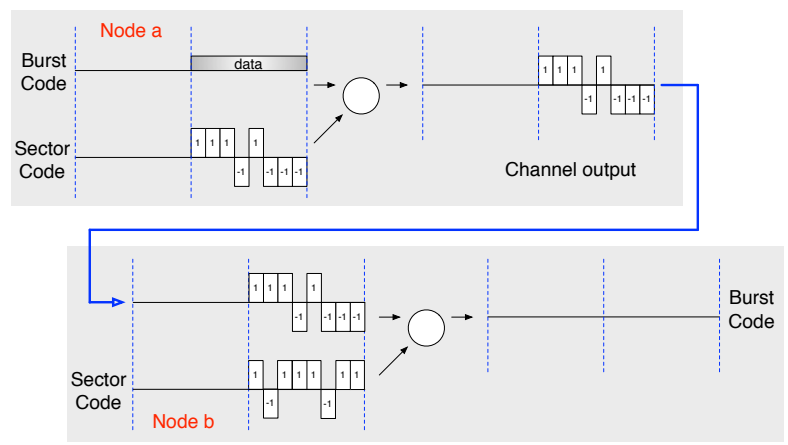

Figure 3: Selection process: two nodes in adjacent sectors

that node $b$ has a burst code equal to [0 0 ] but a sector code equal to [1 $\left.\begin{array}{lllllll}-1 & 1 & 1 & 1 & -1 & 1 & 1\end{array}\right]$ since it is not in the same sector as $a$. We can clearly notice, from Figure 3, that node $b$ does not receive the data sent by node $a$ since the two nodes are using different sector codes. The autocorrelation between the received signal and the sector code of $b$ is equal to zero. In this case, node $b$ continues the selection process.

\section{Performance Evaluation}

In order to evaluate the performances of our proposed broadcast scheme we carried out a set of simulations. We implemented and integrated our broadcast schemes in the SENSE 3.0 simulator. In our simulations setup, we considered nodes following the IEEE 802.11 standard with a channel capacity of $1 \mathrm{Mb} / \mathrm{s}$. We assumed an area of 1000 meters by 1000 meters where we deployed 100 nodes uniformly. We assumed that all the nodes are static. Moreover, we compared our approach with the classical flooding scheme. Finally, as the relay selection criterion, we considered a distancebased cost function, where the main objective was to force the farthest nodes to act as relay nodes.

We start our evaluation by analyzing the performances of our proposed scheme when varying the number of sectors from 1 to 32 . In Figures 4.a, 4.b, 4.c and 4.d, we plot the delivery ratio, the broadcast delay, the number of packet duplications and the energy broadcasting cost respectively. In Figure 4.a, we denote the packet delivery ratio (i.e. the ratio of the number of nodes taht received the packet) for both our optimized broadcast and pure flooding schemes. We study different numbers of sectors: 1, 2, 4, 8, 16 and 32 sectors. It is clear that, with only 1 or 2 sectors, the flooding scheme outperforms our approach. This due to the fact that we do not allow the packet to be propagated in all directions, which greatly limits the number of nodes relaying the packet and thus reduces the performances. However, 


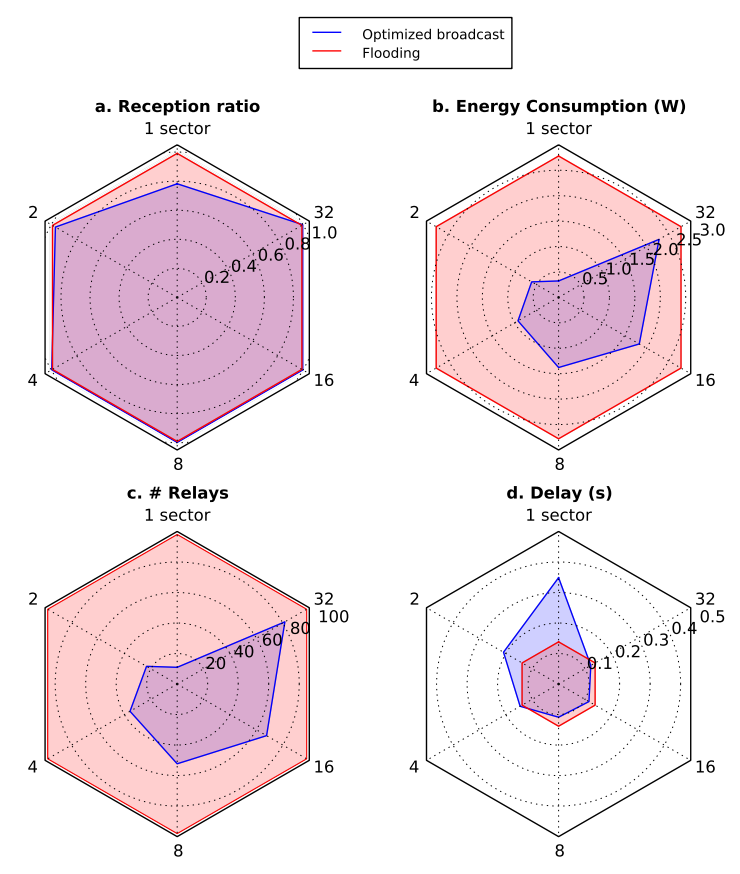

Figure 4: Delivery ratio, delay, number of relays and energy consumption when transmitting only one packet

when we split the communication range in more then 2 sectors our approach gives the same delivery ratio as flooding which is close to $100 \%$. Given that, in Figure 4.d we plot the broadcast delay. By broadcast delay, we mean the delay required for one packet to reach all the network nodes. As expected, and for the same reasons as above, we notice that in the case of 1 or 2 sectors the flooding scheme exhibits smaller delays and outperforms our approach. However, when we increase the number of sectors our approach obtains better delays than flooding.

In order to evaluate the impact of the broadcast algorithm on battery lifetime, we plot the consumed energy for each node for our broadcast and flooding schemes in Figure 4.b. We can see that the average energy consumed by the flooding scheme is much greater than the average energy consumed by our optimized broadcast scheme. Even if we increase the number of sectors to 32, our approach outperforms the flooding scheme. This is confirmed in Figure 4.c, where we plot the number of relays required.

In addition, we analyzed the performances of our scheme when we increase the traffic load. In this case, we fix the number of broadcast sources to $1 \%, 2 \%, 4 \%, 6 \%, 8 \%$ and finally $10 \%$ of the total number of deployed nodes. Also, we consider that each source is generating a CBR packet traffic with an average packet inter arrival time varying from $100 \mathrm{~ms}$ to $400 \mathrm{~ms}$, with 


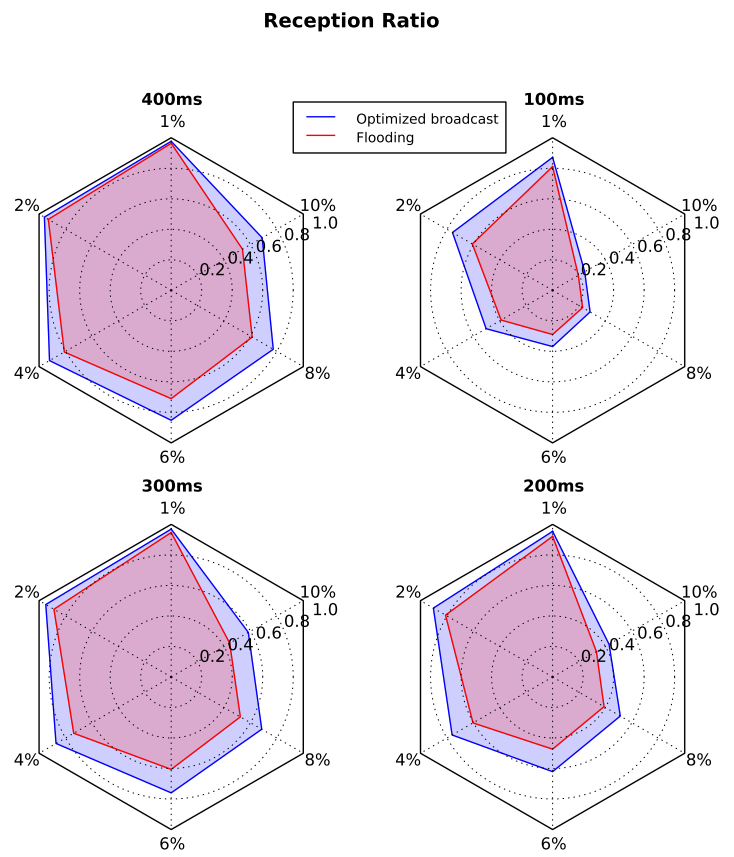

Figure 5: Delivery ratio

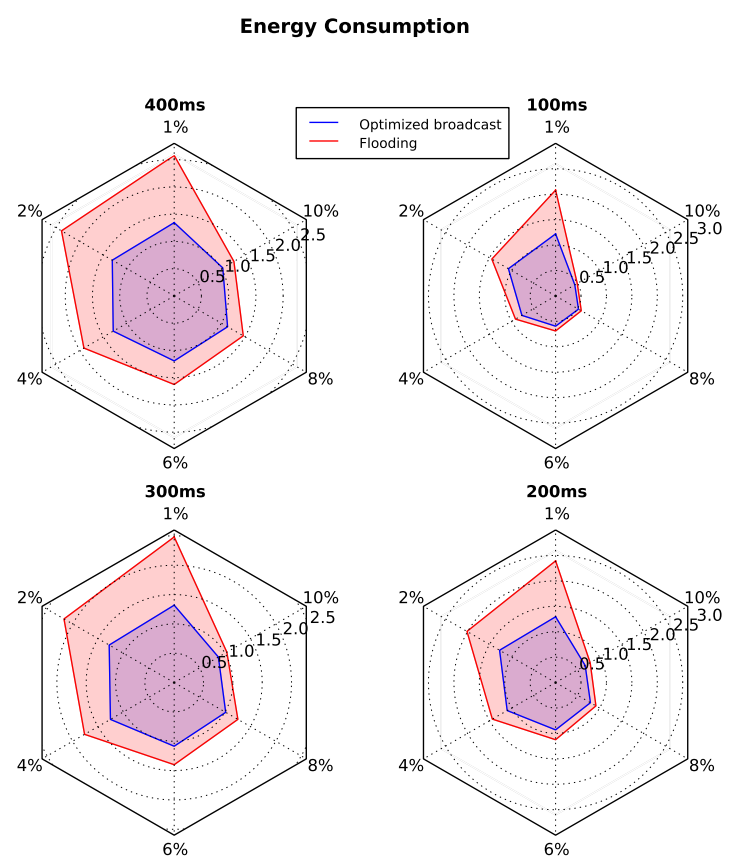

Figure 6: Energy consumption 
a incremental step of $100 \mathrm{~ms}$. As we can observe in Figure 5, the delivery ratio achieved by our proposal consistently outperforms the delivery ratio obtained using flooding. Finally, in Figure 6 we plot the per packet energy consumption. As we can see, the optimized broadcast scheme consumes less energy than the flooding scheme.

\section{Conclusion}

In this paper, we present an efficient broadcast strategy for MANETs. We consider a cost function for packet transmissions and we we study how to determine the best relays for this cost function. Our idea is, as for unicast communications, to use an acknowledgment scheme to select the appropriate relay nodes from all the potential relays within the transmission area. We split the transmission range into several sectors and within each sector, all the nodes that have captured the broadcast packet are required to transmit a short acknowledgment made up of active signaling bursts. This burst code consists of a sequence of intervals of the same length in which a given receiver can either transmit or listen. This acknowledgment phase guarantees that the potential relays that have the highest burst code are selected as relay nodes. Moreover, in order to guarantee that nodes belonging to adjacent sectors do not eliminate each other, we propose that during the transmitting interval (corresponding to 1 in the burst code) each node has to send CDMA orthogonal spreading codes. The code that must be used by each potential relay is selected according to its sector. Finally, we evaluated our approach and we compared it to the classical flooding scheme. The results obtained demonstrate that our proposal outperforms flooding by increasing the delivery ratio and decreasing the number of required relays and thus the energy-cost.

\section{References}

[1] S. Khan, Y. Peng, E. Steinbach, M. Sgroi, and W. Kellerer, "Application-driven cross-layer optimization for video streaming over wireless networks," Communications Magazine, IEEE, vol. 44, no. 1, pp. 122-130, 2006.

[2] Y.-C. Tseng, S.-Y. Ni, Y.-S. Chen, and J.-P. Sheu, "The broadcast storm problem in a mobile ad hoc network," Wirel. Netw., vol. 8, no. 2/3, pp. 153-167, 2002.

[3] H. Lim and C. Kim, "Multicast tree construction and flooding in wireless ad hoc networks," in MSWIM '00: Proceedings of the 3rd ACM international workshop on Modeling, analysis and simulation of wire- 
less and mobile systems. New York, NY, USA: ACM Press, 2000, pp. $61-68$.

[4] W. Peng and X.-C. Lu, "On the reduction of broadcast redundancy in mobile ad hoc networks," in MobiHoc '00: Proceedings of the 1st ACM international symposium on Mobile ad hoc networking $\&$ computing. Piscataway, NJ, USA: IEEE Press, 2000, pp. 129-130.

[5] A. Qayyum, L. Viennot, and A. Laouiti, "Multipoint relaying: An efficient technique for flooding in mobile wireless networks," INRIA, Tech. Rep. Research Report RR-3898, february 2000. [Online]. Available: citeseer.ist.psu.edu/qayyum00multipoint.html

[6] W. Peng and X. Lu, "Ahbp: An efficient broadcast protocol for mobile ad hoc networks," J. Comput. Sci. Technol., vol. 16, no. 2, pp. 153-167, 2001.

[7] B. Williams and T. Camp, "Comparison of broadcasting techniques for mobile ad hoc networks," in MobiHoc '02: Proceedings of the 3rd ACM international symposium on Mobile ad hoc networking $\&$ computing. New York, NY, USA: ACM Press, 2002, pp. 194-205.

[8] J. Macker, "Simplified multicast forwarding for manet." IETF Internet-Draft. March 2007.

[9] B. Blaszczyszyn, A. Laouiti, P. Muhlethaler, and Y. Toor, "Opportunistic broadcast in vanets (ob-van) using active signaling for relays selection," in ITS Telecommunications, 2008. ITST 2008. 8th International Conference on, 2008, pp. 384-389.

[10] P. Jacquet, P. Minet, P. Muhlethaler, and N. Rivierre, "Priority and collision detection with active signaling - the channel access mechanism of hiperlan," Wireless Personal Communications, vol. 4, no. 1, pp. 1125, 1997. 